\title{
A New Method for Ranking Generalized Fuzzy Numbers for Handling Fuzzy Risk Analysis Problems
}

\author{
Jim-Ho Chen Shyi-Ming Chen \\ Department of Computer Science and Information Engineering \\ National Taiwan University of Science and Technology \\ Taipei, Taiwan, R. O. C.
}

\begin{abstract}
In this paper, we present a new method for ranking generalized fuzzy numbers for dealing with fuzzy risk analysis problems. The proposed method considers the defuzzified values, the heights and the spreads of generalized fuzzy numbers, simultaneously, for ranking generalized fuzzy numbers. It gets better ranking results to rank generalized fuzzy numbers than the existing methods. We also apply the proposed method to deal with fuzzy risk analysis problems. The proposed method provides a useful way for handling fuzzy risk analysis problems.
\end{abstract}

Keywords: Defuzzified Values, Fuzzy Risk Analysis, Generalized Fuzzy Numbers, Ranking Order, Standardized Generalized Fuzzy Numbers.

\section{Introduction}

To deal with fuzzy risk analysis problems, experts generally use fuzzy numbers to represent the evaluating value of the risk of each subcomponent made by each manufactory. In order to properly evaluate the risk of components made by manufactories, many methods have been presented for ranking fuzzy numbers [1]-[4], [7]-[15]. Although many methods for ranking fuzzy numbers have been presented, in [3] and [10], Chen et al. pointed out that there are still some drawbacks for the existing methods.

In this paper, in order to overcome the drawbacks of the existing methods, we propose a new method for ranking generalized fuzzy numbers. The proposed method considers the defuzzified values, the heights and the spreads of generalized fuzzy numbers, simultaneously, to rank generalized fuzzy numbers. The experts can set the degree of importance $\alpha$ of the spreads of the fuzzy numbers for ranking fuzzy numbers, where $\alpha=\{0.5,1.5\}$. If the experts consider that the spread is more importance, then they can set the value of $\alpha=1.5$. Otherwise, they can set the value of $\alpha=0.5$. The proposed method gets better ranking results to rank generalized fuzzy numbers than the existing methods. We also apply the proposed method to deal with fuzzy risk analysis problems. The proposed method provides a useful way for handling fuzzy risk analysis problems.

\section{A New Method for Ranking Generalized Fuzzy Numbers}

In 1985, Chen [2] proposed the concept of generalized fuzzy numbers. Let $\tilde{A}$ be a generalized trapezoidal fuzzy number, $\tilde{A}=\left(a_{1}, a_{2}, a_{3}, a_{4} ; w_{\tilde{A}}\right)$, as shown in Fig. 1, where $a_{1}, a_{2}, a_{3}, a_{4}$ are real values, $w_{\tilde{A}}$ denotes the height of the generalized trapezoidal fuzzy number $\tilde{A}$, and $w_{\tilde{A}} \in[0,1]$. If $0 \leq a_{1} \leq a_{2} \leq a_{3} \leq a_{4}$, then $\tilde{A}$ is called a standardized generalized fuzzy number. If $w_{\tilde{A}}=1$, then $\tilde{A}$ is called a normalized fuzzy number and it can be represented as $\tilde{A}=\left(a_{1}, a_{2}, a_{3}, a_{4}\right)$. If $a_{2}=a_{3}$, then $\tilde{A}$ is a triangular fuzzy number. If $a_{1}=a_{2}=a_{3}=a_{4}$, then $\tilde{A}$ is a crisp value.

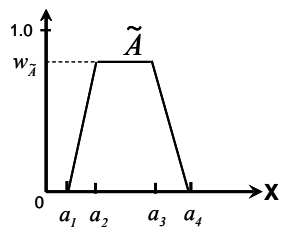

Fig. 1: A generalized trapezoidal fuzzy number $\tilde{A}$

If $\tilde{A}$ is a trapezoidal fuzzy number, i.e., $\tilde{A}=\left(a_{1}, a_{2}, a_{3}, a_{4}\right)$, then the defuzzified value $x_{\tilde{A}}$ of the fuzzy number $\tilde{A}$ can be calculated as follows [6]:

$$
x_{\tilde{A}}=\frac{a_{1}+a_{2}+a_{3}+a_{4}}{4} .
$$

In this section, we present a new method for ranking generalized fuzzy numbers, where we consider three factors for ranking generalized fuzzy numbers, simultaneously, i.e., the defuzzified values of generalized fuzzy numbers, the height of generalized fuzzy numbers, and the spreads of generalized fuzzy numbers. Assume that there are $n$ generalized trapezoidal füzzy numbers $A_{1}, A_{2}, \cdots$, and $A_{n}$ to be ranked, where $A=\left(a_{1}, a_{2}, a_{3}, a_{4} ; w_{\tilde{A}}\right)$ and $1 \leq i \leq$ 
$n$. The proposed method for ranking generalized trapezoidal fuzzy numbers is now presented as follows:

Step 1: Standardize each generalized fuzzy number $\tilde{A}_{i}$ into standardized generalized fuzzy numbers $\tilde{A}_{i}^{*}$, shown as follows:

$$
\begin{aligned}
\tilde{A}_{i}^{*} & =\left(\frac{a_{i 1}}{k}, \frac{a_{i 2}}{k}, \frac{a_{i 3}}{k}, \frac{a_{i 4}}{k} ; w_{\tilde{A}_{i}}\right) \\
& =\left(a_{i 1}^{*}, a_{i 2}^{*}, a_{i 3}^{*}, a_{i 4}^{*} ; w_{\tilde{A}_{i}}\right),
\end{aligned}
$$

where $k$ denotes the maximum value of the universe of discourse and $1 \leq i \leq n$.

Step 2: Calculate the defuzzified value $x_{\tilde{A}_{i}}$ of each standardized generalized fuzzy number $\tilde{A}_{i}^{*}$, shown as follows:

$$
x_{\tilde{A}_{i}^{*}}=\frac{{a_{i 1}}^{*}+a_{i 2}{ }^{*}+a_{i 3}{ }^{*}+a_{i 4}{ }^{*}}{4},
$$

where $1 \leq i \leq n$.

Step 3: Calculate the spread $S T D_{\tilde{\pi}_{*}^{*}}$ of each standardized generalized fuzzy number $\tilde{A}_{i}^{*}$, shown as follows:

$$
S T D_{\tilde{A}_{i}^{*}}=\sqrt{\frac{\sum_{j=1}^{4}\left(a_{i j}{ }^{*}-x_{\tilde{A}_{i}^{*}}\right)^{2}}{4-1}},
$$

where $1 \leq i \leq n$.

Step 4: Calculate the $\operatorname{Score}\left(\tilde{A}_{i}^{*}\right)$ of each standardized generalized fuzzy number $\tilde{A}_{i}^{*}$, shown as follows:

$$
\operatorname{Score}\left(\tilde{A}_{i}^{*}\right)=x_{\tilde{A}_{i}^{*}} \times w_{\tilde{A}_{i}} \times\left(1-\alpha \cdot S T D_{\tilde{A}_{i}^{*}}\right),
$$

where $\alpha$ is a parameter for adjusting the degree of importance of the spreads of the generalized fuzzy numbers and $\alpha \in\{0.5,1.5\}$. If the experts consider that the spread is a more important factor, then they can set the value of $\alpha=1.5$. Otherwise, they can set the value of $\alpha=0.5$. The larger the value of $\operatorname{Score}\left(\tilde{A}_{i}^{*}\right)$, the better the ranking of $\tilde{A}_{i}^{*}$, where $1 \leq i \leq n$.

In the following, we use an example to illustrate the ranking process of the proposed method. Assume that there are two generalized fuzzy numbers $\widetilde{A}=(1,3,4,5 ; 0.6)$ and $\tilde{B}=(2,3,4,5 ; 0.8)$ as shown in Fig. 2(a). Because the maximum value of the universe of discourse $X$ is 5 (i.e., $k=5$ ), based on Eq. (2), the generalized fuzzy numbers of $\tilde{A}$ and $\widetilde{B}$ are transformed into standardized generalized fuzzy numbers $\tilde{A}^{*}$ and $\tilde{B}^{*}$, respectively, where $\tilde{A}^{*}=(0.2,0.6$, $0.8,1.0 ; 0.6)$ and $\tilde{B}^{*}=(0.4,0.6,0.8,1.0 ; 0.8)$ as shown in Fig. 2(b). Then, based on Eq. (3), we can calculate the centers of gravity $x_{\tilde{A}^{*}}$ and $x_{\tilde{B}^{*}}$ of $\tilde{A}^{*}$ and $\tilde{B}^{*}$, respectively, where $x_{\tilde{A}^{*}}=0.65$ and $x_{\tilde{B}^{*}}=0.70$.

Then, based on Eq. (4), we can calculate the spread $S T D_{\tilde{A}^{*}}$ and $S T D_{\tilde{B}^{*}}$ of $\tilde{A}^{*}$ and $\tilde{B}^{*}$, respectively, where $S T D_{\tilde{A}^{*}}=0.3416$ and $S T D_{\tilde{B}^{*}}=0.2582$. Finally, based on
Eq. (5), we can get the scores $\operatorname{Score}\left(\tilde{A}^{*}\right)$ and $\operatorname{Score}\left(\tilde{B}^{*}\right)$ of $\tilde{A}^{*}$ and $\tilde{B}^{*}$, respectively, where

Score $\left(\tilde{A}^{*}\right)=0.65 \times 0.6 \times(1-1.5 \times 0.3416)=0.1902$,

Score $\left(\tilde{B}^{*}\right)=0.7 \times 0.8 \times(1-1.5 \times 0.2582)=0.3431$.

Because $\operatorname{Score}\left(\tilde{B}^{*}\right)>\operatorname{Score}\left(\tilde{A}^{*}\right)$, we can see that the ranking order between the fuzzy numbers $\tilde{A}$ and $\tilde{B}$ is " $B>A$ ".

From this example, we can see that the larger the center of gravity, or the larger the heights, or the smaller the spreads of a fuzzy number, the higher the value of the ranking score of the fuzzy number.
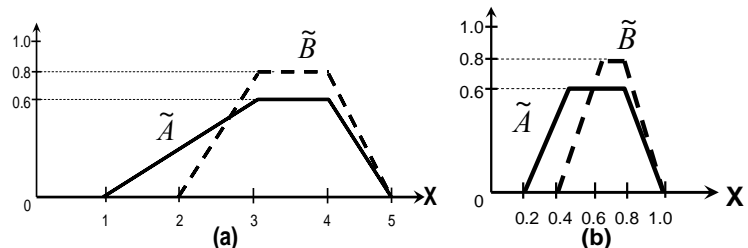

Fig. 2: Generalized fuzzy numbers. (a) Before the standardization process. (b) After the standardization process

\section{Handling Fuzzy Risk Analysis Problems Based on the Proposed Fuzzy Ranking Method}

In this section, we apply the proposed method for ranking generalized fuzzy numbers to deal with fuzzy risk analysis problems, where the evaluating values of fuzzy risk analysis problems are often represented by fuzzy numbers. Assume that there are $n$ manufactories $C_{l}, C_{2}, \ldots$, and $C_{n}$ and assume that component $A_{i}$ made by manufactory $C_{i}$ consists of $p$ sub-components $A_{i l}, A_{i 2}, \cdots$, and $A_{i p}$. The component $A_{i}$ made by manufactory $C_{i}$ is used to analyze the probability of failure $R_{i k}$ of each component $A_{i}$ made by manufactory $C_{i}$, where $1 \leq i \leq \mathrm{n}$. We use two evaluating indices $R_{i k}$ and $W_{i k}$ to evaluate each sub-component $A_{i k}$, where $R_{i k}$ and $W_{i k}$ are generalized fuzzy numbers, $R_{i k}$ denotes the probability of failure of the sub-component $A_{i k}$ of each manufactory $C_{i}, W_{i k}$ denotes the severity of loss of the sub-component $A_{i k}$ of each manufactory $C_{i}, 1 \leq k \leq p$, and $1 \leq i \leq n$. In the following, we briefly review arithmetic operations between fuzzy numbers from [2] and [5]. Assume that there are two generalized $\tilde{A}=\left(a_{1}, a_{2}, a_{3}, a_{4} ; w_{\widetilde{A}}\right)$ and $B=\left(b_{1}, b_{2}, b_{3}, b_{4} ; w_{\widetilde{B}}\right)$, where $a_{1}$, $a_{2}, a_{3}, a_{4}, b_{1}, b_{2}, b_{3}, b_{4}$, are real values, $0 \leq w_{\tilde{A}} \leq 1$, and $0 \leq w_{\tilde{B}} \leq 1$. The arithmetic operations set between $\tilde{A}$ and $\tilde{B}$ are as follows:

Generalized Fuzzy Numbers Addition $\oplus$ :

$$
\begin{aligned}
\tilde{A} \oplus \tilde{B} & =\left(a_{1}, a_{2}, a_{3}, a_{4} ; w_{\tilde{A}}\right) \oplus\left(b_{1}, b_{2}, b_{3}, b_{4} ; w_{\tilde{B}}\right) \\
& =\left(a_{1}+b_{1}, a_{2}+b_{2}, a_{3}+b_{3}, a_{4}+b_{4} ; \min \left(w_{\tilde{A}}, w_{\tilde{B}}\right)\right) .
\end{aligned}
$$

Generalized Fuzzy Numbers Multiplication $\otimes$ :

$\tilde{A} \otimes \widetilde{B}=\left(a_{1}, a_{2}, a_{3}, a_{4} ; w_{\tilde{A}}\right) \otimes\left(b_{1}, b_{2}, b_{3}, b_{4} ; w_{\tilde{B}}\right)$ 


$$
=\left(a_{1} \times b_{1}, a_{2} \times b_{2}, a_{3} \times b_{3}, a_{4} \times b_{4} ; \min \left(w_{\tilde{A}}, w_{\tilde{B}}\right)\right) .
$$

Generalized Fuzzy Numbers Division $\varnothing$ :

$$
\begin{aligned}
\widetilde{A} \varnothing \widetilde{B} & =\left(a_{1}, a_{2}, a_{3}, a_{4} ; w_{\tilde{A}}\right) \varnothing\left(b_{1}, b_{2}, b_{3}, b_{4} ; w_{\tilde{B}}\right) \\
& =\left(a_{1} / b_{4}, a_{2} / b_{3}, a_{3} / b_{2}, a_{4} / b_{1} ; \min \left(w_{\tilde{A}}, w_{\tilde{B}}\right)\right) .
\end{aligned}
$$

The proposed fuzzy risk analysis algorithm is now presented as follows:

Step 1: Based on the fuzzy numbers $R_{i k}$ and $W_{i k}$ of each sub-component $A_{i k}$ of each component $A_{i}$ made by manufactory $C_{i}$, where $1 \leq k \leq p$ and $1 \leq i \leq n$, calculate the probability of failure $R_{i}$ of each component $A_{i}$ made by manufactory $C_{i}$, shown as follows:

$$
\tilde{R}_{i}=\left(\sum_{k=1}^{p} \tilde{R}_{i k} \otimes \tilde{W}_{i k}\right) \varnothing\left(\sum_{k=1}^{p} \tilde{W}_{i k}\right),
$$

where $\tilde{R}_{i}=\left(r_{i 1}, r_{i 2}, r_{i 3}, r_{i 4} ; w_{\tilde{R}_{i}}\right)$ and $1 \leq i \leq n$.

Step 2: Standardize each generalized fuzzy number $R_{i}$ into $R_{i}^{*}$ by Eq. (2), where $1 \leq i \leq n$.

Step 3: Calculate the defuzzified value $\left(x_{\tilde{R}_{i}^{*}}, y_{\tilde{R}_{i}^{*}}\right)$ of each standardized generalized fuzzy number $\tilde{R}_{i}^{*}$ by Eq. (3), where $1 \leq i \leq n$.

Step 4: Calculate the spreads $S T D_{\tilde{R}_{i}^{*}}$ of each standardized generalized fuzzy number $\tilde{R}_{i}^{*}$ by Eq. (4), where $1 \leq i \leq n$.

Stpe 5: Calculate the $\operatorname{Score}\left(\tilde{R}_{i}^{*}\right)$ of each standardized generalized fuzzy number $\tilde{R}_{i}^{*}$ by Eq. (5), where $1 \leq i \leq$ $n$. The larger the value $\operatorname{Score}\left(\tilde{R}_{i}^{*}\right)$, the higher the risk of component $A_{i}$ made by manufactory $C_{i}$, where $1 \leq i$ $\leq n$.

In the following, we use an example shown in [9] to illustrate the fuzzy risk analysis process of the proposed method. Assume that there are three manufactories $C_{1}, C_{2}$ and $C_{3}$ and assume that the components $A_{1}, A_{2}$ and $A_{3}$ are the same product manufactured by different manufactories, where component $A_{i}$ is made by manufactory $C_{i}$ consisting of three sub-components $A_{i 1}, A_{i 2}$ and $A_{i 3}$, where $1 \leq i \leq 3$.

Assume that there are two evaluating items, i.e., the probability of failure $R_{i k}$ and the severity of loss $W_{i k}$, where $R_{i k}$ and $W_{i k}$ are generalized fuzzy numbers, $1 \leq i \leq 3$, and $1 \leq k \leq 3$. Evaluate each sub-component $A_{i k}$ to derive the probability of failure $R_{i}$ of component $A_{i}$ made by manufactory $C_{i}$, where $R_{i k}$ denotes the probability of failure of the sub-component $A_{i k}, W_{i k}$ denotes the severity of loss of the sub-component $A_{i k}$, $1 \leq i \leq 3$ and $1 \leq k \leq 3$. We use a 9-number linguistic term set shown in Table 1 [16] to represent the linguistic terms and their corresponding generalized fuzzy numbers, where the linguistic terms shown in Table 1 are used to represent the linguistic values of the evaluating items $R_{i k}$ and $W_{i k}$ of the sub-components $A_{i 1}, A_{i 2}$ and $A_{i 3}$ made by each manufactory $C_{i}$, where 1 $\leq i \leq 3$, as shown in Table 2 [5].

Table 1. Linguistic Terms and Their Corresponding Generalized Fuzzy Numbers [16]

\begin{tabular}{|c|c|c|c|c|}
\hline Manufactory & Sub-Components & $\begin{array}{l}\text { Linguistic Values of } \\
\text { the Severity of Loss }\end{array}$ & $\begin{array}{c}\text { Linguistic Values of the } \\
\text { Probability of Failure }\end{array}$ & $\begin{array}{l}\text { Degree of Confidence of } \\
\text { the Probability of Failure }\end{array}$ \\
\hline \multirow{3}{*}{$\mathrm{C}_{1}$} & $\mathrm{~A}_{11}$ & $\tilde{W}_{11}=$ Low & $\widetilde{R}_{11}=$ Fairly-Low & $w_{\tilde{R}_{I I}}=0.9$ \\
\hline & $\mathrm{A}_{12}$ & $\tilde{W}_{12}=$ Fairly-High & $\widetilde{R}_{12}=$ Medium & $w_{\widetilde{R}_{l 2}}=0.7$ \\
\hline & $\mathrm{A}_{13}$ & $\tilde{W}_{13}=$ Very-Low & $\tilde{R}_{13}=$ Fairly-High & $w_{\widetilde{R}_{13}}=0.8$ \\
\hline \multirow{3}{*}{$\mathrm{C}_{2}$} & $\mathrm{~A}_{21}$ & $\tilde{W}_{21}=$ Low & $\tilde{R}_{21}=$ Very-High & $w_{\tilde{R}_{2 I}}=0.85$ \\
\hline & $\mathrm{A}_{22}$ & $\tilde{W}_{22}=$ Fairly-High & $\widetilde{R}_{22}=$ Fairly-High & $w_{\tilde{R}_{22}}=0.9$ \\
\hline & $\mathrm{A}_{23}$ & $\tilde{W}_{23}=$ Very-Low & $\tilde{R}_{23}=$ Medium & $w_{\widetilde{R}_{23}}=0.9$ \\
\hline \multirow{3}{*}{$\mathrm{C}_{3}$} & $\mathrm{~A}_{31}$ & $\tilde{W}_{31}=$ Low & $\widetilde{R}_{31}=$ Fairly-Low & $w_{\tilde{R}_{31}}=0.95$ \\
\hline & $\mathrm{A}_{32}$ & $\tilde{W}_{32}=$ Fairly-High & $\tilde{R}_{32}=$ High & $w_{\widetilde{R}_{32}}=0.8$ \\
\hline & $\mathrm{A}_{33}$ & $\tilde{W}_{33}=$ Very-Low & $\tilde{R}_{33}=$ Fairly-High & $w_{\widetilde{R}_{33}}=1.0$ \\
\hline
\end{tabular}

\begin{tabular}{cc}
\hline Linguistic Terms & Generalized Fuzzy Numbers \\
\hline Absolutely-Low & $(0.0,0.0,0.0,0.0 ; 1.0)$ \\
Very-Low & $(0.0,0.0,0.02,0.07 ; 1.0)$ \\
Low & $(0.04,0.10,0.18,0.23 ; 1.0)$ \\
Fairly-Low & $(0.17,0.22,0.36,0.42 ; 1.0)$ \\
Medium & $(0.32,0.41,0.58,0.65 ; 1.0)$ \\
Fairly-High & $(0.58,0.63,0.80,0.86 ; 1.0)$ \\
High & $(0.72,0.78,0.92,0.97 ; 1.0)$ \\
Very-High & $(0.93,0.98,1.0,1.0 ; 1.0)$ \\
Absolutely-High & $(1.0,1.0,1.0,1.0 ; 1.0)$ \\
\hline
\end{tabular}

Table 2. Linguistic Values of the Evaluating Items of the Sub-Components Made by the Manufactories [5]

In Table 2, $w_{\tilde{R}_{k}}$ denotes the degree of confidence of the decision-maker with respect to sub-components $A_{i k}$ made by manufactory $C_{i}$, where $1 \leq i \leq 3$ and $1 \leq k$ $\leq 3$. The process for fuzzy risk analysis is illustrated as follows:

[Step 1] The probability of failure $R_{l}$ of the component $A_{1}$ made by the manufactory $C_{l}$ is calculated by Eq. (9), shown as follows:

$$
\begin{aligned}
\tilde{R}_{1} & =\left[\tilde{R}_{11} \otimes \tilde{W}_{11} \oplus \tilde{R}_{12} \otimes \tilde{W}_{12} \oplus \tilde{R}_{13} \otimes \tilde{W}_{13}\right] \varnothing\left[\tilde{W}_{11} \oplus \tilde{W}_{12} \oplus \tilde{W}_{13}\right] \\
& =(0.1659,0.2803,0.7463,1.1545 ; 0.7) .
\end{aligned}
$$

In the same way, the probability of failure $R_{2}$ of the component $A_{2}$ made by the manufactory $C_{2}$ and the probability of failure $R_{3}$ of the component $A_{3}$ made by the manufactory $C_{3}$ can be calculated by Eq. (9), respectively, where

$R_{2}=(0.3221,0.4949,1.1392,1.6373 ; 0.85)$,

$R_{3}=(0.3659,0.5134,1.1189,1.5984 ; 0.8)$.

[Step 2] Based on Eq. (2), we can transform the generalized fuzzy numbers $R_{l}, R_{2}$ and $R_{\mathcal{Z}}$ into standardized generalized fuzzy numbers $\widetilde{R}_{l}^{*}, R_{2}^{*}$ and $\tilde{R}_{3}^{*}$, respectively, where $\tilde{R}_{l}^{*}=(0.083,0.1402,0.3732,0.5773 ; 0.7)$, 
$\tilde{R}_{2}^{*}=(0.1611,0.2475,0.5696,0.8187 ; 0.85)$,

$\widetilde{R}_{3}^{*}=(0.183,0.2567,0.5595,0.7992 ; 0.8)$.

[Step 3] Based on Eq. (3), we can calculate the defuzzified values $x_{\tilde{R}_{I}^{*}}, x_{\tilde{R}_{2}^{*}}$ and $x_{\tilde{R}_{L}^{*}}$ of the standardized generalized fuzzy numbers $R_{1}^{*}, \tilde{R}_{2}^{*}$ and $\tilde{R}_{3}^{*}$, respectively, where $x_{\tilde{R}_{1}^{*}}=0.29, x_{\tilde{R}_{2}^{*}}=0.45$ and $x_{\tilde{R}_{3}^{*}}=0.45$.

[Step 4] Based on Eq. (4), we can calculate the spreads $S T D_{\tilde{R}_{1}^{*}}, S T D_{\tilde{R}_{2}^{*}}$ and $S T D_{\tilde{R}_{3}^{*}}$ of the standardized generalized fuzzy numbers $\tilde{R}_{1}^{*}, \tilde{R}_{2}^{*}$ and $\tilde{R}_{3}^{*}$, respectively, where $S T D_{\tilde{R}_{1}^{*}}=0.2271, S T D_{\tilde{R}_{2}^{*}}=0.3026$ and $S T D_{\widetilde{R}_{3}^{*}}=0.2844$.

[Step 5] Based on Eq. (5), we can calculate the ranking values $\operatorname{Score}\left(\widetilde{R}_{1}^{*}\right), \operatorname{Score}\left(\tilde{R}_{2}^{*}\right)$ and $\operatorname{Score}\left({\widetilde{\widetilde{R}_{3}^{*}}}_{\tilde{R}^{*}}\right)$ of the standardized generalized fuzzy numbers $\widetilde{\sim}_{1}^{*}$, $\widetilde{R}_{2}^{*}$ and $\widetilde{R}_{3}^{*}$, respectively, where

$\operatorname{Score}\left(\tilde{R}_{l}^{*}\right)=0.29 \times 0.7 \times(1-1.5 \times 0.2271)=0.1338$, $\operatorname{Score}\left(\tilde{R}_{2}^{*}\right)=0.45 \times 0.85 \times(1-1.5 \times 0.3026)=0.2089$, $\operatorname{Score}\left(\tilde{R}_{3}^{*}\right)=0.45 \times 0.8 \times(1-1.5 \times 0.2844)=0.2064$.

Because $\operatorname{Score}\left(\tilde{R}_{2}^{*}\right)>\operatorname{Score}\left(\tilde{R}_{3}^{*}\right)>\operatorname{Score}\left(\tilde{R}_{1}^{*}\right)$, the ranking order of the risk of the manufactories $C_{1}, C_{2}$ and $C_{3}$ is " $C_{2}>C_{3}>C_{1}$ ". It coincides with the result of the one presented in [5].

\section{Conclusions}

In this paper, we have presented a new method for ranking generalized fuzzy numbers. The proposed method considers three factors for ranking generalized fuzzy numbers, simultaneously, i.e., the defuzzified values, the height and the spreads of generalized fuzzy numbers. The proposed method gets better ranking results than Chen-and-Chen's method [3] and Chuand-Tsao's method [10]. Moreover, we also apply the proposed ranking method to deal with fuzzy risk analysis problems. The proposed method provides a useful way for handling fuzzy risk analysis problems.

\section{Acknowledgements}

This work was supported in part by the National Science Council, Republic of China, under Grant NSC 94-2213-E-011-005.

\section{References}

[1] J. R. Chang, C. H. Cheng, and C. Y. Kao, "Conceptual procedure for ranking fuzzy numbers based on adaptive two-dimensions dominance," Soft Computing, vol. 10, no. 2, pp. 94-103, 2006.

[2] S. H. Chen, "Ranking fuzzy numbers with maximizing set and minimizing set," Fuzzy Sets and Systems, vol. 17, no. 2, pp. 113-129, 1985.
[3] S. J. Chen and S. M. Chen, “A new method for handling the fuzzy ranking and the defuzzification problems," Proceedings of the 2000 Eighth National Conference on Fuzzy Theory and Its Applications, Taipei, Taiwan, Republic of China, 2000.

[4] S. J. Chen and C. L. Hwang, Fuzzy Multiple Attribute Decision Making. Berlin, Heidelberg: Springer-Verlag, 1992.

[5] S. J. Chen, New Information Fusion and Information Aggregation Method for Handling Multicriteria Fuzzy Decision-Making Problems, Ph.D. Dissertation, National Taiwan University of Science and Technology, Taipei, Taiwan, R. O. C., 2004.

[6] S. M. Chen, W. H. Hsiao, and Y. J. Horng, “A knowledge-based method for fuzzy query processing for document retrieval," Cybernetics and Systems, vol. 28, no. 1, pp. 99-119, 1997.

[7] L. H. Chen and H. W. Lu, "An approximate approach for ranking fuzzy numbers based on left and right dominance," Computers and Mathematics with Applications, vol. 41, no. 12, pp. 1589-1602, 2001.

[8] L. H. Chen and H. W. Lu, "The preference order of fuzzy numbers,” Computers and Mathematics with Applications, vol. 44, no. 10, pp. 1455-1465, 2002.

[9] C. H. Cheng, “A new approach for ranking fuzzy numbers by distance method," Fuzzy Sets and Systems, vol. 95, no. 3, pp. 307-317, 1998.

[10] T. C. Chu and C. T. Tsao, "Ranking fuzzy numbers with an area between the centroid point and original point," Computers and Mathematics with Applications, vol. 43, no. 1, pp. 111-117, 2002.

[11] M. Delgado, J. L. Verdegay, and M. A. Villa, “A procedure for ranking fuzzy numbers using fuzzy relations," Fuzzy Sets and Systems, vol. 26, no. 1, pp. 49-62, 1988.

[12] D. Dubois and H. Prade, "Ranking of fuzzy numbers in the setting of possibility theory," Information Sciences, vol. 30, no. 3, pp. 183-224, 1983.

[13] H. W. Lu, A study of fuzzy numbers ranking methods, M.S. Thesis, Department of Industrial Management, National Cheng Kung University, Taiwan, Republic of China, 2001.

[14] H. W. Lu and C.B. Wang, “An index for ranking fuzzy numbers by belief feature,” Information and Management Sciences, vol. 16, no. 3, pp. 5770, 2005.

[15] M. Nojavan and M. Ghazanfari, "A fuzzy ranking method by desirability index," Journal of Intelligent and Fuzzy Systems, vol. 17, no. 1, pp. 27-34, 2006.

[16] W. R. Zhang, Knowledge Representation Using Linguistic Fuzzy Relations, Ph. D. Dissertation, University of South Carolina, U. S. A., 1986. 